Arq. Bras. Med. Vet. Zootec., v.67, n.2, p.381-390, 2015

\title{
Influência do parasitismo intestinal sobre os parâmetros hematológicos e de líquido peritoneal em equinos de tração
}

\author{
[Influence of intestinal parasitism on peritoneal fluid and hematological \\ parameters in horses used for traction] \\ C.L. Lhamas $^{1}$, C.A. Duarte ${ }^{2}$, I. Lübeck ${ }^{2}$, M.N.G. Silva ${ }^{2}$, C.T. Leite ${ }^{1}$, E.P. Pereira ${ }^{2}$, \\ K. Roschildt ${ }^{2}$, F. Bernardon ${ }^{2}$ \\ ${ }^{1}$ Programa de pós-graduação - Universidade Federal de Santa Maria - UFSM - Santa Maria, RS \\ ${ }^{2}$ Universidade Federal do Pampa - Unipampa - Uruguaiana, RS
}

\begin{abstract}
RESUMO
O objetivo do presente estudo foi avaliar a influência do parasitismo intestinal crônico sobre parâmetros hematológicos e de líquido peritoneal por meio da comparação dessas características em equinos naturalmente parasitados e após administração de anti-helmíntico. Utilizaram-se 21 cavalos de tração urbana, entre dois e 19 anos, sem raça definida e com resultado de exame parasitológico superior a 300 ovos por grama de fezes. Foi realizada avaliação física e coleta de fezes, de líquido peritoneal e de sangue em dois momentos do experimento (D0 e D15), sendo efetuado tratamento antiparasitário no D0. No fluido peritoneal foram avaliadas características físicas, bioquímicas, bem como contagem de células nucleadas (CTCN) e diferenciação celular. No sangue foram determinados valores eritrocitários, leucocitários, proteínas plasmáticas totais, glicose e fibrinogênio plasmáticos, além de fosfatase alcalina (FA) sérica. A análise dos parâmetros avaliados não demonstrou diferença significativa entre animais parasitados e após administração de anti-helmíntico, exceto para valores de CTCN, contagem de neutrófilos segmentados e grau de turbidez do líquido peritoneal. As médias se mantiveram dentro dos intervalos de referência, com exceção da CTCN do líquido peritoneal no D0. No líquido peritoneal, houve predomínio de neutrófilos segmentados, seguidos por macrófagos, linfócitos e eosinófilos em ambos os momentos de avaliação. Observaram-se tendência do quadro eritrocitário em manter-se próximo aos limites inferiores e leve leucocitose no D0. A infecção parasitária nos animais estudados foi predominantemente moderada, o que oferece poucos riscos clínicos. Nessas condições, pode-se afirmar que a CTCN, a contagem absoluta de neutrófilos segmentados e o grau de turbidez do líquido peritoneal são influenciados e podem ser considerados ferramentas diagnósticas e prognósticas úteis nas parasitoses intestinais crônicas.
\end{abstract}

Palavras-chave: equídeos, parasitismo intestinal, avaliação hematológica, avaliação parasitológica, líquido peritoneal

\section{ABSTRACT}

The aim of this study was to evaluate the influence of chronic intestinal parasitism on hematological parameters and peritoneal fluid. It was done by comparing these features in horses used for traction naturally parasitized and after the administration of anthelmintic. Twenty-one horses, between two and nineteen years of age, of mixed breed and with results of parasitological examination of more than 300 eggs per gram of feces were studied. Physical assessment and samples of feces were conducted, as well as blood and peritoneal fluid in the two phases of the experiment (DO and D15). Antiparasitic treatment in DO has also been done. The peritoneal fluid was evaluated for physical and biochemical features, and also total count of nucleated cells (TCNC) and cell differentiation. The blood was determined for erythrocyte, leukocyte, plasma total protein, glucose and plasma fibrinogen, and alkaline phosphatase $(A L P)$ levels. The analysis of these parameters showed no significant difference between parasitized animals and after administration of anthelmintic except for TCNC values, segmented neutrophil count

Recebido em 15 de fevereiro de 2014

Aceito em 9 de janeiro de 2015

E-mail: cibelelhamas@hotmail.com 
and degree of turbidity in peritoneal fluid. The averages remained within the reference ranges, except the $T C N C$ in the peritoneal fluid in DO. In the peritoneal fluid there was a predominance of segmented neutrophils, followed by macrophages, lymphocytes and eosinophils in both time points. A trend was observed in erythrocyte frame to keep close to the lower limits and mild leukocytosis in D0. Parasitic infection of the animals studied was predominantly moderate, which offered minimal clinical risks. After that, it can be affirmed that the TCNC, absolute segmented neutrophil count and targeted degree of turbidity in peritoneal fluid are influenced and can be considered useful diagnostic and prognostic tools in chronic intestinal parasitism.

Keywords: equidae, intestinal parasitism, hematological evaluation, parasitological evaluation, peritoneal fluid

\section{INTRODUÇÃO}

As afecções gastrintestinais compreendem um dos problemas mais frequentes em equinos, sendo destacada a síndrome cólica causada por obstruções, rupturas e deslocamentos intestinais (Pierezan, 2009). Os cavalos de tração também são muito afetados pelas afecções do sistema digestório, porém a principal causa é a parasitose (Andrade et al., 2009).

A relação existente entre altas infecções parasitárias e alterações hematológicas é amplamente estudada. Nessas condições, são relatadas anemia e eosinofilia (Duncan e Pirie, 1975; Hubert et al., 2004). No entanto, existe ainda escassez de informações sobre a interpretação da análise do líquido peritoneal nas doenças abdominais, incluindo as parasitoses gastrintestinais.

O fluido peritoneal reflete as alterações que ocorrem na superfície mesotelial da cavidade abdominal, e o estudo de suas características físico-químicas e citológicas permite avaliar a condição da cavidade em equinos com lesões abdominais (Malark et al., 1992). A inflamação do peritônio pode ocorrer em resposta a uma variedade de estímulos tanto infecciosos (bactérias, vírus, fungos e parasitos), quanto não infecciosos (traumas, agentes químicos e neoplasias). Garma-Aviña (1998) sugeriu a migração de células inflamatórias para o líquido peritoneal em condições de injúrias causadas por helmintos.

Dessa forma, o objetivo do presente estudo foi avaliar a influência do parasitismo intestinal crônico sobre parâmetros hematológicos e de líquido peritoneal por meio da comparação dessas características em equinos de tração naturalmente parasitados e após a administração de anti-helmíntico.

\section{MATERIAL E MÉTODOS}

O protocolo experimental utilizado no presente estudo foi aprovado pelo Comitê de Ética para o Uso de Animais, sob registro CEUA da Universidade Federal do Pampa no . 008-2012. Foram utilizados 21 equinos de tração urbana (13 machos e oito fêmeas não prenhes), sem raça definida, com idades entre dois e 19 anos (média de 10,5 $\pm 8,5$ anos de idade) e peso corporal médio de $317,5 \pm 32,9 \mathrm{~kg}$. Os cavalos que compuseram o grupo experimental foram aqueles que apresentaram resultado coprológico com contagem igual ou superior a 300 ovos por grama de fezes (OPG) no dia zero (D0) do experimento e sem alterações clínicas detectáveis no momento das coletas.

Os animais foram submetidos à coleta de fezes, de líquido peritoneal e de sangue em dois momentos do experimento (dia zero e dia 15). No primeiro dia de coleta (D0) foi realizada a vermifugação com composto à base de ivermectina $\left(0,2 \mathrm{mg} \mathrm{kg}^{-1}\right)$ e praziquantel $(2,5 \mathrm{mg}$ $\mathrm{kg}^{-1}$ ) (Ivermectina, Ouro Fino Gel Composto 9,6 GR, Brasil).

O diagnóstico parasitológico foi realizado mediante a coleta das fezes diretamente da ampola retal, sendo o material acondicionado em caixa isotérmica com gelo. A análise quantitativa foi realizada por meio da técnica de Gordon e Whitlock (1939). No dia 15 (D15), a mesma avaliação foi efetuada para confirmação da eficácia do tratamento antiparasitário.

Para coleta do líquido peritoneal, foi realizada abdominocentese segundo a técnica descrita por Peloso e Cohen (2012). A 
amostra coletada era fracionada em frascos estéreis contendo anticoagulante EDTA (ácido etilenodiaminotetracetico-potássico) para contagem total de células nucleadas (CTCN), proteína total $\left(\left[\mathrm{PT}_{\text {ref }}\right]\right)$ e densidade; frascos sem anticoagulante para avaliação física e da atividade da fosfatase alcalina (FA); frasco contendo fluoreto de sódio/EDTA para mensuração da glicose; e outro frasco com citrato trissódico anidro $(3,2 \%)$ para determinação do fibrinogênio.

O fluido peritoneal foi avaliado mediante análises física, celular e bioquímica. $\mathrm{Na}$ avaliação física, após homogeneização das amostras, foram observados coloração, grau de turbidez e densidade. A coloração foi classificada em amarelo-palha, alaranjada, avermelhada, amarelo-ouro e transparente. $\mathrm{O}$ grau de turbidez foi categorizado em límpido, ligeiramente turvo e turvo. A densidade e a concentração das proteínas totais $\left(\left[\mathrm{PT}_{\mathrm{ref}}\right]\right)$ foram realizadas em refratômetro manual (Refratômetro manual portátil (proteína/densidade), Instrutherm, Brasil).

A avaliação bioquímica do líquido peritoneal incluiu a mensuração da glicose (Kit Glicose, Laborlab, Brasil) e da atividade da fosfatase alcalina (FA) (Kit Fosfatase Alcalina, Laborclin, Brasil) com o auxílio de um conjunto de reagentes e leituras espectrofotométricas (Espectrofotômetro UV visível, modelo LGS 53). A avaliação do fibrinogênio foi feita com auxílio de um kit coagulométrico (Fibrinogen Hemostasis, Labtest, Brasil).

A avaliação celular foi realizada por meio da contagem total de células nucleadas (CTCN) e da contagem diferencial. A CTCN foi obtida pelo método de contagem manual por câmara ou hemocitômetro de Neubauer, utilizando-se a metodologia descrita por Schalm (1970) e Willard et al. (1999). A contagem diferencial de células nucleadas foi realizada usando-se "squashs" das amostras corados pelo método do corante hematológico rápido (panótico). Posteriormente, as preparações citoscópicas foram examinadas por microscopia óptica, em aumento de 1000x (imersão).

Para coleta das amostras de sangue, foi realizada venopunção da jugular externa e coleta de aproximadamente $20 \mathrm{~mL}$ de sangue em frascos estéreis com e sem EDTA, frascos contendo fluoreto de sódio/EDTA e frascos contendo citrato trissódico anidro $(3,2 \%)$. A realização da avaliação hematológica foi feita por meio dos métodos descritos por Schalm (1970), incluindo leucometria global, hematimetria pelo método do hemocitômetro e determinação do volume globular pelo método do micro-hematócrito. A contagem diferencial de células foi determinada no esfregaço sanguíneo após coloração pelo método do corante hematológico rápido e revisão microscópica, incluindo pesquisa de hemoprotozoários. Os valores de volume corpuscular médio (VCM) e de concentração de hemoglobina corpuscular média (CHCM) foram calculados segundo as fórmulas clássicas recomendadas por Jain (1993).

O soro foi imediatamente separado da porção sanguínea e foram realizados testes para atividade de FA. As proteínas plasmáticas totais $\left(\left[\mathrm{PPT}_{\mathrm{ref}}\right]\right)$ foram determinadas por refratometria. O fibrinogênio plasmático foi avaliado por meio do método descrito por Clauss (1957), e a glicose plasmática e a atividade da FA foram mensuradas com o auxílio de um conjunto de reagentes e leituras espectrofotométricas.

As análises estatísticas foram realizadas com auxílio do pacote estatístico IBM SPSS Statistics, versão 19.0. Por apresentarem distribuição normal, comprovada pelo teste de Shapiro-Wilk, os dados foram contrastados mediante o teste $t$ de Student pareado, para comparação entre dias. Os dados que não assumiram distribuições gaussianas foram submetidos ao teste não paramétrico pareado de Wilcoxon. As variáveis qualitativas (coloração e turbidez do líquido peritoneal) foram contrastadas pelo teste de quiquadrado. Os dados foram apresentados como média e \pm desvio-padrão da média. Para todas as análises foi considerado o nível de significância de $5 \%$.

\section{RESULTADOS}

Nos exames fecais, foi observada a infecção por parasitos das ordens Strongylida e Ascaridida identificados pela morfologia dos ovos (Hoffmann, 1987). Os valores de OPG mínimos e máximos verificados no D0 foram 300 e 1.900, respectivamente, com média aritmética de 796,4 $\pm 468,8$ e mediana 800,0 . Foram encontrados sete $(33,3 \%)$ animais com valores inferiores a 500 
OPG, nove $(42,8 \%)$ com carga parasitária entre 500 e 1.000 OPG, e cinco $(23,8 \%)$ com um elevado parasitismo, apresentando valores superiores a 1.000 OPG. A média de OPG verificada em animais com até três anos de idade foi de $1.356 \pm 512,9$; animais entre três e 12 anos de idade tiveram média de 661,5 $\pm 263,5$; e animais acima de 12 anos apresentaram média de OPG de 675 638,7 . Houve eliminação total dos ovos nos animais no D15 do experimento com todos os resultados zerados.

Quanto às variáveis qualitativas do líquido peritoneal, a maioria dos animais apresentou fluido de coloração amarelo-palha nos D0 e D15. $\mathrm{Na}$ distribuição das porcentagens para o grau de turbidez, houve predominância do aspecto límpido após a administração do vermífugo (Tab. $1)$.

Tabela 1. Variações percentuais da coloração e turbidez do líquido peritoneal em 21 equinos de tração naturalmente parasitados (dia zero) e após vermifugação (dia 15)

\begin{tabular}{ccc}
\hline Momentos/Variáveis & Dia zero & Dia 15 \\
\hline Amarelo-palha & $57,9 \mathrm{~A}$ & $73,7 \mathrm{~A}$ \\
Alaranjado & $21,1 \mathrm{~B}$ & $15,8 \mathrm{~B}$ \\
Avermelhado & $10,5 \mathrm{C}$ & $5,3 \mathrm{C}$ \\
Amarelo-ouro & $5,3 \mathrm{D}$ & $0 \mathrm{D}$ \\
Transparente & $5,3 \mathrm{E}$ & $5,3 \mathrm{E}$ \\
\hline Límpido & $26,3 \mathrm{~F}$ & $63,2 \mathrm{G}$ \\
Ligeiramente turvo & $36,8 \mathrm{H}$ & $10,5 \mathrm{I}$ \\
Turvo & $36,8 \mathrm{~J}$ & $26,3 \mathrm{~J}$ \\
\hline
\end{tabular}

Letras diferentes indicam que houve diferenças $(\mathrm{P} \leq 0,05)$ entre os dias zero e 15 .

Quando as variáveis de coloração foram divididas como normal e alterada, assumindo-se como normais os padrões amarelo-palha, amarelo-ouro e transparente, e alteradas as demais colorações, observaram-se $68,4 \%$ de coloração normal nos animais parasitados contrastando com 78,9\% da mesma coloração após vermifugação. Não houve diferença significativa na comparação entre esses valores. Por outro lado, quando as variáveis de turbidez foram classificadas como normal e alterada, assumindo-se como normal o padrão límpido, e alterados os demais padrões, observaram-se $26,3 \%$ das amostras normais no D0 em contraste com $63,2 \%$ no D15. A comparação entre esses valores indicou a presença de diferença significativa com valor de $\mathrm{P}=0,022$.
Nas análises física e bioquímica do líquido peritoneal (Tab. 2), foi realizada a comparação entre os valores de densidade, da $\left[\mathrm{PT}_{\text {ref }}\right]$, do fibrinogênio, da glicose e da atividade da FA entre os D0 e D15. Os valores de densidade no líquido peritoneal não variaram significativamente. Da mesma forma, não houve diferenças entre as médias dos parâmetros bioquímicos citados em ambos os momentos de coleta. Durante a mensuração de fibrinogênio, não foi observada coagulação pelo método utilizado. Esse resultado indicou que a concentração dessa proteína esteve abaixo de $100 \mathrm{mg} / \mathrm{dL}$ em todas as amostras avaliadas nos dois momentos de coleta.

Tabela 2. Valores médios, desvios-padrão e valor de $\mathrm{P}$ das análises física e bioquímica do líquido peritoneal em 21 equinos de tração naturalmente parasitados (dia zero) e após vermifugação (dia 15)

\begin{tabular}{ccccc}
\hline $\begin{array}{c}\text { Variáveis/Médias e } \\
\text { desvios-padrão }\end{array}$ & Densidade & $\begin{array}{c}\text { Proteína total } \\
(\mathrm{g} / \mathrm{dL})\end{array}$ & Glicose $(\mathrm{mg} / \mathrm{dL})$ & $\begin{array}{c}\text { Fosfatase alcalina } \\
(\mathrm{UI} / \mathrm{L})\end{array}$ \\
\hline Dia zero & $1.015 \pm 0,003$ & $2,381 \pm 0,6$ & $91,8 \pm 13,1$ & $11,5 \pm 19,6$ \\
Dia 15 & $1.013 \pm 0,002$ & $2,237 \pm 0,4$ & $98,2 \pm 20,8$ & $19,7 \pm 47,5$ \\
Valor de P & 0,124 & 0,206 & 0,268 & 0,346 \\
\hline
\end{tabular}

Valor de $\mathrm{P}=$ grau de significância estabelecido em $5 \%(\mathrm{P} \leq 0,05)$. Valores de $\mathrm{P}$ maiores que 0,05 não apresentam diferença significativa. 
Durante a avaliação citológica do líquido peritoneal (Tab. 3 e 4), foi observado que a média encontrada no D0 para a CTCN foi de $2.855 \pm 4.623$ células/ $\mu \mathrm{L}$ e no D15 foi de $1.273 \pm$ 960 células $/ \mu \mathrm{L}$. Houve diferença significativa entre os dois momentos de coleta tanto para
CTCN quanto para neutrófilos segmentados. Por outro lado, não houve diferenças para as demais linhagens celulares. Basófilos e células mesoteliais não foram observados à avaliação citoscópica nos dois momentos de coleta.

Tabela 3. Valores médios e desvios-padrão de valores relativos de leucócitos (\%) no líquido peritoneal em 21 equinos de tração naturalmente parasitados (dia zero) e após vermifugação (dia 15)

\begin{tabular}{ccccc}
\hline $\begin{array}{c}\text { Variáveis/Médias e } \\
\text { desvios-padrão }\end{array}$ & $\begin{array}{c}\text { Neutrófilos } \\
\text { segmentados }(\%)\end{array}$ & Macrófagos (\%) & Linfócitos (\%) & $\begin{array}{c}\text { Eosinófilos } \\
(\%)\end{array}$ \\
\hline Dia zero & $64,8 \pm 21,4$ & $31,3 \pm 20,1$ & $2,2 \pm 2,5$ & $1,5 \pm 2,7$ \\
Dia 15 & $60,5 \pm 17,1$ & $32,0 \pm 14,4$ & $5,1 \pm 5,6$ & $2,0 \pm 5,5$ \\
\hline
\end{tabular}

Tabela 4. Valores médios, desvios-padrão e valor de $\mathrm{P}$ de contagem total de células nucleadas e dos valores absolutos de leucócitos $(/ \mu \mathrm{L})$ no líquido peritoneal em 21 equinos de tração naturalmente parasitados (dia zero) e após vermifugação (dia 15)

\begin{tabular}{cccccc}
\hline $\begin{array}{c}\text { Variáveis/Média } \\
\text { s e desvios- } \\
\text { padrão }\end{array}$ & $\begin{array}{c}\text { CTCN* } \\
(/ \mu \mathrm{L})\end{array}$ & $\begin{array}{c}\text { Neutrófilos } \\
\text { segmentados } \\
(/ \mu \mathrm{L})\end{array}$ & $\begin{array}{c}\text { Macrófagos } \\
(/ \mu \mathrm{L})\end{array}$ & $\begin{array}{c}\text { Linfócitos } \\
(/ \mu \mathrm{L})\end{array}$ & $\begin{array}{c}\text { Eosinófilos } \\
(/ \mu \mathrm{L})\end{array}$ \\
\hline Dia zero & $2.855 \pm 4.623$ & $1.671 \pm 2.282$ & $1.043 \pm 2.310$ & $34,3 \pm 55,2$ & $103 \pm 396$ \\
Dia 15 & $1.273 \pm 960$ & $776 \pm 637$ & $411 \pm 352$ & $52,8 \pm 46,4$ & $22,7 \pm 45,2$ \\
Valor de P & 0,0102 & 0,0112 & 0,0611 & 0,1909 & 0,5049 \\
\hline
\end{tabular}

*CTCN $=$ Contagem total de células nucleadas.

Valor de $\mathrm{P}=$ grau de significância estabelecido em $5 \%(\mathrm{P} \leq 0,05)$. Valores de $\mathrm{P}$ maiores que 0,05 não apresentam diferença significativa.

A avaliação dos parâmetros hematimétricos demonstrou que não houve alterações significativas comparando-se os animais no D0 e no D15 (Tab. 5). À avaliação citoscópica, não foi observada presença de hemoprotozoários nos esfregaços sanguíneos dos animais estudados.

Tabela 5. Valores médios, desvios-padrão e valor de $\mathrm{P}$ dos valores hematimétricos em 21 equinos de tração naturalmente parasitados (dia zero) e após vermifugação (dia 15)

\begin{tabular}{cccccc}
\hline $\begin{array}{c}\text { Variáveis/Média } \\
\text { s e desvios- } \\
\text { padrão }\end{array}$ & $\begin{array}{c}\text { Hematócrito } \\
(\%)\end{array}$ & $\begin{array}{c}\text { Hemácias } \\
(/ \mu \mathrm{L})\end{array}$ & $\begin{array}{c}\text { Hemoglobina } \\
(\mathrm{g} / \mathrm{dL})\end{array}$ & VCM (fL) & CHCM $(\%)$ \\
\hline Dia zero & $30,4 \pm 4,8$ & $7,095 \pm 1,9$ & $10,1 \pm 1,6$ & $43,8 \pm 11,5$ & $33,1 \pm 0,13$ \\
Dia 15 & $30,1 \pm 3,5$ & $6,781 \pm 1,3$ & $10,0 \pm 1,1$ & $45,7 \pm 7,7$ & $33,1 \pm 0,08$ \\
Valor de P & 0,803 & 0,4439 & 0,834 & 0,577 & 0,727
\end{tabular}

Valor de $\mathrm{P}=$ grau de significância estabelecido em $5 \%(\mathrm{P} \leq 0,05)$. Valores de $\mathrm{P}$ maiores que 0,05 não apresentam diferença significativa.

Na avaliação dos parâmetros leucométricos, não foi observada diferença significativa na concentração de leucócitos entre os dois momentos de coleta. Também não houve diferença significativa para as linhagens celulares quando comparadas entre os animais parasitados e após vermifugação (Tab. 6).
Durante avaliação da bioquímica sérica, da proteína plasmática total e do fibrinogênio plasmático (Tab. 7), a mensuração desses valores não demonstrou diferenças significativas na comparação dos valores obtidos entre os animais do D0 e D15. 
Tabela 6. Valores médios, desvios-padrão e valor de $\mathrm{P}$ dos valores absolutos de leucócitos plasmáticos $(/ \mu \mathrm{L})$ em 21 equinos de tração naturalmente parasitados (dia zero) e após vermifugação (dia 15)

\begin{tabular}{ccccccc}
\hline $\begin{array}{c}\text { Variáveis/Média } \\
\text { s e desvios- } \\
\text { padrão }\end{array}$ & $\begin{array}{c}\text { Leucócitos } \\
\text { totais } \\
\left(\mathrm{x} 10^{3} / \mu \mathrm{L}\right)\end{array}$ & $\begin{array}{c}\text { Segmentado } \\
\mathrm{s}\end{array}$ & $\begin{array}{c}\text { Linfócitos } \\
(/ \mu \mathrm{L})\end{array}$ & $\begin{array}{c}\text { Monócitos } \\
(/ \mu \mathrm{L})\end{array}$ & $\begin{array}{c}\text { Eosinófilos } \\
(/ \mu \mathrm{L})\end{array}$ & $\begin{array}{c}\text { Basófilos } \\
(/ \mu \mathrm{L})\end{array}$ \\
\hline Dia zero & $12.124 \pm 3.648$ & $7.874 \pm 2.928$ & $3.197 \pm 1.684$ & $584,2 \pm 426,2$ & $351,6 \pm 319,1$ & $127,3 \pm 197,8$ \\
Dia 15 & $11.538 \pm 2.860$ & $7.242 \pm 2.895$ & $3.078 \pm 1.103$ & $640,7 \pm 584,8$ & $315,1 \pm 273,7$ & $90,8 \pm 114$ \\
Valor de P & 0,4521 & 0,3252 & 0,6797 & 0,6911 & 0,579 & 0,5883 \\
\hline
\end{tabular}

Valor de $\mathrm{P}=$ grau de significância estabelecido em $5 \%(\mathrm{P} \leq 0,05)$. Valores de $\mathrm{P}$ maiores que 0,05 não apresentam diferença significativa.

Tabela 7. Valores médios, desvios-padrão e valor de $\mathrm{P}$ dos valores de bioquímica sérica e proteínas plasmáticas totais em 21 equinos de tração naturalmente parasitados (dia zero) e após vermifugação (dia 15)

\begin{tabular}{ccccc}
\hline $\begin{array}{c}\text { Variáveis/Médias } \\
\text { e desvios-padrão }\end{array}$ & $\begin{array}{c}\text { Fosfatase alcalina } \\
(\mathrm{UI} / \mathrm{L})\end{array}$ & $\begin{array}{c}\text { Proteínas } \\
\text { plasmáticas totais } \\
(\mathrm{g} / \mathrm{dL})\end{array}$ & $\begin{array}{c}\text { Fibrinogênio } \\
(\mathrm{mg} / \mathrm{dL})\end{array}$ & $\begin{array}{c}\text { Glicose } \\
(\mathrm{mg} / \mathrm{dL})\end{array}$ \\
\hline Dia zero & $160,7 \pm 48,5$ & $8,840 \pm 0,42$ & $309,5 \pm 184,1$ & $84,0 \pm 14,4$ \\
Dia 15 & $144,3 \pm 53,2$ & $8,829 \pm 0,46$ & $257,1 \pm 112,1$ & $88,5 \pm 20,8$ \\
Valor de P & 0,276 & 0,774 & 0,192 & 0,443 \\
\hline
\end{tabular}

Valor de $\mathrm{P}=$ grau de significância estabelecido em $5 \%(\mathrm{P} \leq 0,05)$. Valores de $\mathrm{P}$ maiores que 0,05 não apresentam diferença significativa.

\section{DISCUSSÃO}

A infecção parasitária foi moderada na maioria dos animais estudados, portanto não acarretou alterações significativas nas funções vitais ao se compararem animais parasitados e após a vermifugação. $\mathrm{O}$ parasitismo moderado corrobora resultado do trabalho de Schuster et al. (2011) e difere do encontrado por Andrade et al. (2009), no qual animais com menor carga parasitária (até 500 OPG) foram predominantes. A faixa etária dos animais avaliados reforça a relação existente entre animais jovens e o elevado parasitismo (Tizard, 2009), pois observou-se que a maior média de OPG foi verificada em cavalos com até três anos de idade.

Pela morfologia dos ovos, foram identificados parasitos das ordens Strongylida e Ascaridida. Matthews (2011) cita que os equinos são mais susceptíveis a esses vermes, os quais possuem alta capacidade de proliferação e resistência no meio ambiente, podendo sobreviver por longos períodos nas pastagens.

A avaliação do exame físico do líquido peritoneal demonstrou que a maioria dos animais apresentou a coloração amarelo-palha tanto no D0 quanto no D15. McCraw e Slocombe (1985) relataram líquido peritoneal de coloração marrom-escura em animais infectados com larvas de Strongylus equinus. Tais parasitos causam migração peritoneal e esse seria o principal motivo de alteração pronunciada na coloração das amostras.

Os animais que apresentaram coloração alaranjada e avermelhada poderiam ter tido a amostra contaminada com sangue no momento da coleta, situação que não é incomum (Malark et al., 1992; Alleman, 2003). Entretanto, Malark et al. (1992) observaram que não ocorre um aumento significativo na [PT] e na CTCN de amostras contaminadas. Em casos em que não há contaminação, a presença de hemácias e neutrófilos fagocitados no líquido peritoneal pode levar à suspeita de hemorragia em alças intestinais, causada por lesão de vasos sanguíneos devido à migração parasitária (Marinkovic et al., 2009). Foi verificada presença de leucofagia e eritrofagocitose mesmo em animais com líquido peritoneal de coloração amarelo-palha, o que indica hemorragia na cavidade abdominal, provavelmente não recente.

A distribuição das porcentagens para o grau de turbidez foi de $26,3 \%$ para o padrão límpido e $36,8 \%$ para ambos os padrões ligeiramente turvo e turvo no D0, entretanto o aspecto límpido predominou no D15 (63,2\%). Em um estudo avaliando quatro cavalos com intussuscepções cecais associadas à ciatostomíase larval, a 
avaliação do líquido peritoneal de dois animais demonstrou características de exsudato com base em aspectos de turbidez e CTCN. Intussuscepções intestinais associadas a ciatostomíneos são raramente relatadas, sendo Anoplocephala perfoliata reconhecido como um dos principais causadores desse distúrbio (Mair et al., 2000). Ciatostomíase larval e outras doenças causadas por estrongilídeos são responsáveis por danos na parede intestinal na fase de migração larval. Ambas as condições podem provocar lesões intestinais e, consequentemente, levar a alterações no exame do fluido peritoneal.

Os valores de densidade, assim como os bioquímicos, não apresentaram diferenças estatísticas entre os dias experimentais. Contudo, as médias da $\left[\mathrm{PT}_{\text {ref }}\right]$ apresentaram-se maiores do que o valor de referência descrito por Alleman (2003) e de acordo com o observado por Nelson (1979), que cita valores de até $2,8 \mathrm{~g} / \mathrm{dL}$. Qualquer condição que comprometa a integridade da parede intestinal, a exemplo do que ocorre nas parasitoses gastrintestinais, pode levar a uma alteração vascular que favorece o extravasamento de elementos plasmáticos para o líquido peritoneal. Contudo, o aumento da [PT] no fluido peritoneal é mais comumente observado em condições inflamatórias agudas da cavidade (Faria et al., 1999).

O aumento da atividade da FA no líquido peritoneal sugere desvitalização do intestino delgado de equinos com lesões abdominais (Jain, 1993). As médias das concentrações de FA no líquido peritoneal no presente estudo permaneceram dentro dos valores de referência e não houve diferença significativa entre os dois momentos de coleta. Entretanto, foi observado valor aumentado em um animal no D0. Valente et al. (2009) verificaram que a inflamação da cavidade abdominal em animais submetidos a enterorrafias foi evidenciada precocemente com a avaliação de FA no líquido peritoneal. Segundo os autores, a mensuração dessa enzima foi útil no acompanhamento da resolução do processo inflamatório intra-abdominal.

Durante avaliação citoscópica do fluido peritoneal desse animal, foi verificada presença de granulações tóxicas e fagocitose em neutrófilos. Neutrófilos com alterações tóxicas são comumente associados a infecções bacterianas graves (Alleman, 2003), portanto os demais exames complementares devem ser avaliados de forma conjunta para verificação de alterações sistêmicas e investigação das causas dessas alterações. Visto que esse cavalo apresentou alta atividade de FA no líquido peritoneal e leve leucocitose por neutrofilia, acompanhamento clínico foi exigido nessa situação, pois poderia indicar processo infeccioso agudo.

As lesões causadas pela infecção parasitária podem ser uma das causas de peritonite (Davis, 2003), e a mensuração da [glicose] no líquido peritoneal com o conhecimento da [glicose] plasmática é importante nesse diagnóstico (Alleman, 2003). Como observado nas Tab. 2 e 7, a [glicose] no líquido peritoneal encontrava-se maior do que a [glicose] plasmática com valores de $\mathrm{P}=0,004$ no $\mathrm{D} 0$ e de $\mathrm{P}=0,0003$ no D15. A ocorrência de diferença significativa entre as médias de [glicose] no líquido peritoneal e [glicose] plasmática é esperada em situações fisiológicas (Alleman, 2003). A investigação de características como CTCN e [PT] deve ser também considerada para que a peritonite seja diagnosticada (Davis, 2003).

A observação de diferença significativa da CTCN entre animais parasitados e após administração de vermífugo indica que o parasitismo intestinal pode ser uma causa de migração de células inflamatórias para a cavidade peritoneal. Uma efusão cavitária é considerada normal ou transudato simples quando possui CTCN de até 1.500 células $/ \mu \mathrm{L}$ (Alleman, 2003). A média encontrada pode classificar as efusões do D0 como transudato modificado. Ao se avaliarem dois animais neste ensaio com valores elevados e discrepantes de CTCN, pôde-se inferir que esses resultados decorreram de uma fase de migração larval. Por outro lado, no D15, a média da CTCN encontrouse dentro do padrão de transudato simples, indicando ausência do estímulo inflamatório.

Em relação às linhagens celulares observadas, houve diferença estatística na comparação da contagem absoluta de neutrófilos segmentados entre os dois momentos experimentais. A maior população desse tipo celular no D0 pode ser indicativa de processo inflamatório agudo (Alleman, 2003). Houve predominância de neutrófilos segmentados no D0, seguidos por 
macrófagos, linfócitos e eosinófilos. No D15, a mesma proporção foi verificada à contagem relativa (Tab. 3). Esses achados se assemelham aos observados por Neves et al. (2000), que avaliaram características do líquido peritoneal em equinos sadios. A maior contagem absoluta de eosinófilos no D0 em relação ao D15 foi atribuída à presença de valores discrepantes, tendo sido observado em um animal contagem desse tipo celular igual a $1.800 \mathrm{eos} / \mu \mathrm{L}$. Esse achado pode ser devido à infestação parasitária, corroborando Garma-Aviña (1998).

A avaliação do hemograma demonstrou que, quando comparados aos valores de referência (Jain, 1993), há uma tendência do quadro eritrocitário em se manter próximo aos limites inferiores de normalidade nos dois momentos de coleta. A presença de anemia nos animais deste trabalho pode estar relacionada tanto à deficiência de nutrientes provocada por alimentação de baixa qualidade, como demonstrado por Silva Filho et al. (2004), quanto ao parasitismo apresentado por grande parte deles. Eritropoiese defeituosa por problemas na síntese do heme e hemorragia crônica constitui o mecanismo de instalação da anemia nas respectivas causas (Fighera e Graça, 2011).

Os parâmetros de hemograma corroboram os encontrados por Reichmann et al. (2001), em que se verificou tendência à ocorrência de anemia, e divergem dos encontrados por Sartori Filho et al. (1993) e Andrade et al. (2009), em que não foram observadas alterações hematológicas associadas à infecção natural por estrongilídeos. Provavelmente a divergência dos resultados citados seja decorrente das diferentes respostas imunológicas relacionadas com a dose infectante, gerando uma ação não tão intensa do parasito sobre o hospedeiro nas infecções naturais.

Em relação ao quadro leucocitário, foi observada tendência à leucocitose nos dois momentos de avaliação, sendo que houve leve leucocitose no D0. Leucocitose por neutrofilia também foi observada por Reichmann et al. (2001).

Apesar de alguns autores relatarem aumento na concentração de leucócitos no início da infecção parasitária (Duncan e Pirie, 1975), não foi observada diferença significativa entre os dois momentos de coleta. É provável que o grau de infecção detectado no D0 do experimento não ofereça riscos aos animais, pois a média de OPG indicou parasitismo moderado. Mesmo esses animais estando expostos a condições higiênicosanitárias inadequadas, a carga parasitária verificada, em sua maioria, manteve-se entre discreta a moderada, sugerindo equilíbrio na relação hospedeiro-parasito.

Não foram observadas diferenças significativas para os valores de eosinófilos, e as médias tanto do D0 quanto do D15 permaneceram dentro do intervalo de referência (Jain, 1993). Esses achados reforçam a hipótese de que o estímulo parasitário a que esses animais estavam submetidos não era suficiente para alterar de forma proeminente os achados leucométricos. Infecções experimentais maciças puras por $S$. vulgaris (Duncan e Pirie, 1975) geraram quadro de eosinofilia por terem ocorrido particularmente como consequência da migração dos parasitos em fase larval.

As outras linhagens celulares mantiveram-se dentro dos intervalos de referência, não havendo diferenças significativas entre animais parasitados naturalmente e após a vermifugação. Esses resultados foram semelhantes aos observados por Sartori Filho et al. (1993), que não encontraram diferenças significativas no leucograma entre animais parasitados e após tratamento com anti-helmíntico. Da mesma forma, assemelham-se aos achados por Reichmann et al. (2001), os quais observaram leve leucocitose por neutrofilia e valores normais de linfócitos e eosinófilos.

No que tange aos valores de proteínas totais, notou-se que as médias da $\left[\mathrm{PPT}_{\text {ref }}\right]$, em ambos os momentos, permaneceram próximas ao limite superior dos intervalos de referência citados (Jain, 1993). O aumento da proteína plasmática pode ocorrer na desidratação, devido à perda de líquido, e na estimulação da resposta imune, devido ao aumento de imunoglobulinas (Tizard, 2009).

É mais provável que o discreto aumento observado nos animais deste ensaio seja devido principalmente à desidratação leve e, secundariamente, ao estímulo da resposta imune. A premissa de que a ingestão hídrica era inadequada em muitos dos animais estudados e de que, portanto, alguns estavam levemente desidratados pode ser a hipótese mais provável 
para justificar a maior concentração da proteína plasmática.

A análise conjunta dos resultados desta investigação permite inferir que equinos naturalmente parasitados possuem uma relação parasito-hospedeiro simbiótica, possivelmente devido à constante exposição aos agentes parasitários. Sob essas circunstâncias, é possível que o hospedeiro elabore uma resposta mais efetiva contra os parasitos e, consequentemente, altere de forma menos pronunciada seus parâmetros fisiológicos. Contudo, pôde-se verificar que mesmo um estímulo parasitário crônico e moderado foi capaz de alterar a CTCN e a contagem absoluta de neutrófilos segmentados no líquido peritoneal, assim como o grau de turbidez.

\section{CONCLUSÕES}

O parasitismo intestinal natural crônico influenciou diretamente parâmetros importantes na avaliação do líquido peritoneal, entretanto essa influência não foi verificada nos parâmetros hematológicos. As alterações observadas no líquido peritoneal possibilitam afirmar que o parasitismo nas condições estudadas provocou um grau de inflamação local e que esses parâmetros podem ser considerados ferramentas diagnósticas e de acompanhamento da integridade dos órgãos abdominais nas infecções parasitárias crônicas.

\section{AGRADECIMENTOS}

Aos professores Dr. Márcio Machado Costa e Dra $^{\mathrm{a}}$. Mirela Noro, pelo auxílio nas análises estatísticas e pela participação técnica no desenvolvimento deste estudo; à Coordenação de Aperfeiçoamento de Pessoal de Nível Superior (Capes), pela concessão da bolsa de mestrado.

\section{REFERÊNCIAS}

ALLEMAN, A.R. Abdominal, thoracic, and pericardial effusions. Vet. Clin. Small Anim., v.33, p.89-118, 2003.

ANDRADE, R.L.F.S.; SOBRAL, J.C.; SILVA, K.M.G. Avaliação clínica, hematológica e parasitária em equinos de tração na cidade de Aracaju, Sergipe. Acta Vet. Bras., v.3, p.138-142, 2009.
CLAUSS, A. Rapid physiological coagulation method in determination of fibrinogen. Acta Haematol., v.17, p.237-246, 1957.

DAVIS, J.L. Treatment of peritonitis. Vet. Clin. N. Am. - Equine., v.19, p.765-778, 2003.

DUNCAN, J.L.; PIRIE, H.M. The pathogenesis of single experimental infections with Strongylus vulgaris in foals. Res. Vet. Sci., v.18, p.82-93, 1975.

FARIA, E.P.; MARQUES, A.P.; ALVES, G.E.S. Características celulares e bioquímicas do líquido peritoneal de equinos submetidos à peritonite experimental. Arq. Bras. Med. Vet. Zootec., v.51, p.335-344, 1999.

FIGHERA, R.A.; GRAÇA, D.L. Sistema hematopoiético, In: SANTOS, R.L.; ALESSI, A.C. (Ed.), Patologia Veterinária. São Paulo: Roca. 2011. p.337-422.

GARMA-AVIÑA, A. Cytology of 100 samples of abdominal fluid from 100 horses with abdominal disease. Equine Vet. J., v.30, p.435444, 1998.

GORDON, H.M.; WHITLOCK, H.V. A new technique for counting nematode eggs in sheep faeces. J. Counc. Sci. Ind. Res., v.12, p.50-52, 1939.

HOFFMANN, R.P. Diagnóstico Parasitismo Veterinário. Sulina: Porto Alegre, 1987. 156p.

HUBERT, J.D.; SEAHORN, T.L.; KLEI T.R. et al. Clinical sign and hematologic, cytokine, and plasma nitric oxide alterations in response to Strongylus vulgaris infection in helminth-naïve ponies. Can. J. Vet. Res., v.68, p.193-200, 2004.

JAIN, N.C. Essentials of Veterinary Hematology. Lea and Febiger: Philadelphia, 1993. 417p.

MARINKOVI'C, D.; ALEKSI'CKOVACEVI'C, S.; KRSTI'C, V.; KNEZEVI'C, M. Morphological findings in the cranial mesenteric artery of horses with verminous arteritis. Acta Vet.-Beograd., v.59, p.231-241, 2009.

MAIR, T.S.; SUTTON, D.G.; LOVE, S. Caecocaecal and caecocolic intussusceptions associated with larval cyathostomosis in four young horses. Equine Vet. J., v.32, p.77-80, 2000. 
MALARK, J.A.; PEYTON, L.C.; GALVIN, M.J. Effects of blood contamination on equine peritoneal fluid analysis. J. Am. Vet. Med. Assoc., v.201, p.1545-1556, 1992.

MATTHEWS, J.B. Facing the threat of equine parasitic disease. Equine Vet. J., v.43, p.126-132, 2011.

MCCRAW, B.M.; SLOCOMBE, J.O.D. Strongylus equinus: development and pathological effects in the equine host. Can. J. Comp. Med., v.49, p.372-383, 1985.

NELSON, A.W. Analysis of equine peritoneal fluid. Vet. Clin. N. Am.-Large., v.1, p.267-274, 1979.

NEVES, M.M.; MARQUES JR, A.P.; ALVES, G.E.S.; FARIA, E.P. Valores referenciais da análise do líquido peritoneal de equinos sadios. Cienc. Rural, v.30, p.809-811, 2000.

PELOSO, J.G.; COHEN, N.D. Use of serial measurements of peritoneal fluid lactate concentration to identify strangulating intestinal lesions in referred horses with signs of colic. $J$. Am. Vet. Med. Assoc., v.240, p.1208-1217, 2012.

PIEREZAN, F. Prevalência das doenças de equinos no Rio Grande do Sul. 2009. 163p. Dissertação (Mestrado) - Curso de PósGraduação em Medicina Veterinária, Universidade Federal de Santa Maria, Santa Maria.

REICHMANN, P.; LISBOA, J.A.N.; BALARIN, M.R.S.; PEREIRA, A.B.L. Valores hematológicos em equinos naturalmente infectados por estrongilídeos. Semina: Cienc. Agrárias, v.22, p.179-181, 2001.
SARTORI FILHO, R.; AMARANTE, A.E.T.; OLIVEIRA, M.R. Efeito de medicações antihelmínticas com ivermectin e fenbendazole em equinos: exames coprológicos e hematológicos. Rev. Bras. Parasitol. Vet., v.2, p.61-64, 1993.

SCHALM, O.W. Veterinary Hematology. 2. ed. Lea and Febiger: Philadelphia, 664p., 1970.

SCHUSTER, A.B.G.; MARCHIORI, M.; BERNE, M.E.A. et al. Perfil parasitológico dos cavalos de carroça da cidade de Pelotas-RS atendidos durante o período de abril de 2010 a junho de 2011. In: Congresso de Iniciação Científica e III Mostra Científica da UFPel, 20., 2011. Anais... Pelotas, RS., (Resumo) 2011.

SILVA FILHO, J.M.; PALHARES, M.S.; MARANHÃO, R.P.A. et al. Manejo alimentar dos animais de tração da Regional Pampulha, Belo Horizonte. In: Congresso Brasileiro de Extensão Universitária, 2., 2004. Anais... Belo Horizonte, MG., p.1-6, 2004.

TIZARD, I.R. Imunidade adquirida a parasitas. In: Ibid. (Ed.), Imunologia Veterinária: uma introdução., 8.ed. Rio de Janeiro: Saunders Elsevier, 2009. p.312-329.

VALENTE, P.P.; CATTELAN, J.W.; SANTANA, A.E. et al. Concentrações de fibrinogênio plasmático, fosfatase alcalina sérica e do fibrinogênio e fosfatase alcalina no fluido peritoneal de equinos submetidos à enterorrafias aposicional e invaginante no cólon descendente. N. Animalium., v.1, p.95-106, 2009.

WILLARD, M.D.; TVEDTEN, H.; TURNWALD, G.H. Small animal clinical diagnosis by laboratory methods. 3. ed. W.B. Saunders: Philadelphia, 1999. 395p 